\title{
Time-dependent wave front propagation simulation of a hard $x$-ray split-and-delay unit: Towards a measurement of the temporal coherence properties of $x$-ray free electron lasers
}

\author{
S. Roling and H. Zacharias \\ Physikalisches Institut, Westfälische Wilhelms-Universität, 48149 Münster, Germany \\ L. Samoylova, H. Sinn, and Th. Tschentscher \\ European XFEL GmbH, 22761 Hamburg, Germany \\ O. Chubar \\ Brookhaven National Laboratory, Upton, New York 11973, USA \\ A. Buzmakov \\ Shubnikov Institute of Crystallography RAS, 119333 Moscow, Russia \\ E. Schneidmiller and M. V. Yurkov \\ Deutsches Elektronen-Synchrotron, 22603 Hamburg, Germany \\ F. Siewert \\ Helmholtz-Zentrum Berlin für Materialien und Energie, 12489 Berlin, Germany \\ S. Braun and P. Gawlitza \\ Fraunhofer IWS, 01277 Dresden, Germany \\ (Received 4 August 2014; published 18 November 2014)
}

\begin{abstract}
For the European X-ray free electron laser (XFEL) a split-and-delay unit based on geometrical wavefront beam splitting and multilayer mirrors is built which covers the range of photon energies from $5 \mathrm{keV}$ up to $20 \mathrm{keV}$. Maximum delays between $\Delta \tau= \pm 2.5 \mathrm{ps}$ at $h \nu=20 \mathrm{keV}$ and up to $\Delta \tau= \pm 23$ ps at $h \nu=5 \mathrm{keV}$ will be possible. Time-dependent wave-optics simulations have been performed by means of Synchrotron Radiation Workshop software for XFEL pulses at $h \nu=5 \mathrm{keV}$. The XFEL radiation was simulated using results of time-dependent simulations applying the self-amplified spontaneous emission code FAST. Main features of the optical layout, including diffraction on the beam splitter edge and optics imperfections measured with a nanometer optic component measuring machine slope measuring profiler, were taken into account. The impact of these effects on the characterization of the temporal properties of XFEL pulses is analyzed. An approach based on fast Fourier transformation allows for the evaluation of the temporal coherence despite large wavefront distortions caused by the optics imperfections. In this way, the fringes resulting from time-dependent two-beam interference can be filtered and evaluated yielding a coherence time of $\tau_{c}=0.187 \mathrm{fs}$ (HWHM) for real, nonperfect mirrors, while for ideal mirrors a coherence time of $\tau_{c}=0.191 \mathrm{fs}$ (HWHM) is expected.
\end{abstract}

DOI: 10.1103/PhysRevSTAB.17.110705

PACS numbers: 42.25.Kb, 41.60.Cr, 41.50.+h, 07.85.Fv

\section{INTRODUCTION}

Among the light sources available in the hard x-ray regime free-electron laser (FEL) radiation generated by self-amplified spontaneous emission (SASE) provides widely tunable ultrashort light pulses with unprecedented pulse energies, coherence properties and a well-defined wavefront. Besides the already operating LCLS at the

Published by the American Physical Society under the terms of the Creative Commons Attribution 3.0 License. Further distribution of this work must maintain attribution to the author(s) and the published article's title, journal citation, and DOI.
SLAC National Accelerator Laboratory (USA) [1] and SACLA in Japan [2] the European $\mathrm{x}$-ray free electron laser (XFEL) is now under construction in Hamburg (Germany). With electron bunches accelerated to energies of up to $17.5 \mathrm{GeV}$ the machine will provide photon energies between $h \nu=3 \mathrm{keV}$ and $h \nu=24 \mathrm{keV}$ at the undulator sources SASE1 and SASE2. Pulse energies of presumably $E_{\text {pulse }} \approx 2 \mathrm{~mJ}$ and a pulse duration on the order of a few femtoseconds up to a few hundred femtoseconds [3] are expected. In the burst mode very high repetition rates of 2700 pulses at $4.5 \mathrm{MHz}$ per burst at a repetition rate of $10 \mathrm{~Hz}$ will be possible, due to superconducting accelerators. 
One of the most outstanding features of SASE radiation is the high degree of temporal and spatial coherence compared to synchrotron radiation, the other widely tunable source of hard x-ray radiation. In combination with the subnanometer wavelengths single-shot diffraction imaging of nanoscale objects will be possible [4]. Two correlated pulses, separated in the femtosecond regime, enable sequential diffractive imaging [5] and pave the way towards the recording of a molecular movie with subatomic resolution. However, in particular the temporal coherence of the pulses of a SASE FEL is not as perfect as that of the radiation of conventional lasers. Consequently, a measurement of the temporal coherence properties is mandatory not only for evaluating the results of diffractive imaging experiments but also for an understanding and optimization of the SASE process. Thus, in recent years great effort has been devoted to the investigation of the coherence properties of free electron lasers [6-9]. A measurement of the temporal properties of the FEL pulses, like temporal coherence and pulse duration [10], can be performed by using two jitter-free pulse replicas that can be temporally delayed with respect to each other. These replicas can be generated in a split-and-delay unit (SDU) by geometric wavefront beam splitting. Two SDUs of this kind are now operational in the extreme ultraviolet (XUV) and soft $\mathrm{x}$-ray spectral regime at beam lines BL2 [11] and PG2 [12] at FLASH. An SDU for photon energies of $500 \mathrm{eV}<h \nu<$ $2000 \mathrm{eV}$ is available at the atomic, molecular and optical science beam line at the LCLS [13]. As opposed to a classical Michelson interferometer that makes use of an amplitude beam splitter, the coherence properties are measured by overlapping two different points $r_{1}$ and $r_{2}$ of the beam if a wavefront beam splitter is utilized. Thus, as discussed in Ref. [8], a measurement of the temporal coherence is always influenced by the spatial coherence properties of the FEL. This influence is negligible if the points $r_{1}$ and $r_{2}$ are close to each other. In the XUV and soft X-ray spectral regime an amplitude beam splitter can be realized by means of a thin membrane coated with multilayers [14]. Recently, a Michelson interferometer for XUV radiation at $\lambda=$ $13.5 \mathrm{~nm}$ has been developed that utilizes such an amplitude beam splitter [15]. With this device the temporal coherence properties of an FEL can be measured without being influenced by a finite spatial coherence. However, due to the utilization of multilayers only a single wavelength can be transmitted. An approach to generate two pulse replicas in the hard x-ray regime is the use of perfect crystals in Bragg and Laue geometry, which has been realized in the SDUs presented in Refs. [16,17]. However, due to the spectral selectivity of such crystals the spectra of the FEL pulses are monochromatized and consequently the coherence properties are enhanced. This is desired for some experiments but a measurement of the original coherence properties of the FEL pulses is not possible with these devices.

Besides a characterization of the FEL pulse properties, the most essential application of SDUs is their employment for jitter-free pump/probe experiments. Particularly, with the SDU at beam line BL2 at FLASH using XUV pumpprobe spectroscopy the ionization dynamics in expanding clusters [18] and the ultrafast heating of dense cryogenic hydrogen [19] has been investigated.

The conservation of the properties of the FEL pulses is one of the most demanding challenges in the design of $\mathrm{x}$-ray beam lines and instrumentation components $[20,21]$. In particular, it is indispensable to minimize the wavefront distortions caused by mirror imperfections such as slope and height errors. Especially in the hard x-ray regime such wavefront distortions are of importance because the magnitude of the polishing errors approaches the scale of the wavelengths as

$$
\Delta w=2 \Delta h \cdot \sin \theta,
$$

where $\Delta h$ is the residual surface height error and $\theta$ is the grazing incidence angle. The level of wavefront disturbances that can be tolerated also depends on optics layout (distances from the optics to the source and the experiment). The longer the distance is to the experiment, the wider is the spatial frequency range resulting in specklelike wavefront distortions $[20,22]$. The first $800 \mathrm{~mm}$ long offset mirror of the SASE2 beam line with $\Delta h=2 \mathrm{~nm}$ is located at about $300 \mathrm{~m}$ from the source and $600 \mathrm{~m}$ from the experiment. The mirrors in XFEL beam lines are irradiated at grazing incidence angles accounting to $\theta=3.6 \mathrm{mrad}=$ $0.21^{\circ}$ at $h \nu=5 \mathrm{keV}$, which corresponds to a wavelength of $\lambda \approx 0.25 \mathrm{~nm}$. The wavefront distortion caused by the offset mirror accounts to $\Delta w=0.056 \lambda$ and the relevant spatial wavelengths of the surface profile range from 0.5 to $200 \mathrm{~mm}$.

The novel hard $\mathrm{x}$-ray SDU will be integrated in the SASE2 undulator beam line and thus will enable temporally resolved investigations at the high energy density (HED) experiment $[23,24]$. Foremost, it will enable timeresolved $\mathrm{x}$-ray pump/x-ray probe experiments $[18,19]$ as well as sequential diffractive imaging [5] on a femtosecond to picosecond time scale. Another important purpose of this instrument will be a measurement of the temporal coherence properties of hard $x$-ray pulses of the SASE2 undulator by means of a linear autocorrelation [8]. In an SDU much steeper reflecting angles, on the order of 10 to $70 \mathrm{mrad}$, are necessary to provide the experiment with a sufficient delay in the picosecond range. In the hard x-ray regime single layer coatings do not show a sufficient reflectance for such steep angles. Therefore, $\mathrm{Mo} / \mathrm{B}_{4} \mathrm{C}, \mathrm{Ni} / \mathrm{B}_{4} \mathrm{C}$ and $\mathrm{W} / \mathrm{B}_{4} \mathrm{C}$ multilayer Bragg coatings that cover the photon energy range from $h \nu=5 \mathrm{keV}$ to $20 \mathrm{keV}$ are applied to the $\mathrm{Si}$ substrates. The wavefront beam splitter in the SDU will then be irradiated at a Bragg angle of $\theta=64 \mathrm{mrad}=3.6^{\circ}$ for a wavelength of $\lambda=0.25 \mathrm{~nm}$. The distance to the experiment is $z=130 \mathrm{~m}$. The shorter distances from the mirror to the experiment and the steeper incident angle 
reduce the relevant spatial frequency range that allows to tolerate surface height errors despite of larger local absolute wavefront distortions scaled with $\sin \theta$. In this study the influence of these inevitable wavefront distortions and a possible approach to handle them is discussed. The simulation of the propagation of the hard x-ray SASE pulses through the SDU is performed by means of Synchrotron Radiation Workshop (SRW) software $[25,26]$ and Wave Propagator (WPG) framework [27]. As input XFEL radiation obtained from results of time-dependent simulations with the SASE code FAST [28] is used.

\section{DESIGN OF THE HARD X-RAY SDU}

The optical layout of the SDU for the HED experiment at the SASE2 undulator of the European XFEL is based on a point symmetric optical concept and geometrical wavefront beam splitting. Multilayer Bragg coatings of $\mathrm{Mo} / \mathrm{B}_{4} \mathrm{C}$, $\mathrm{Ni} / \mathrm{B}_{4} \mathrm{C}$ and $\mathrm{W} / \mathrm{B}_{4} \mathrm{C}$ permit larger grazing angles between $\theta=64 \mathrm{mrad}=3.6^{\circ}$ at $h \nu=5 \mathrm{keV}$ and $\theta=10 \mathrm{mrad}=$ $0.57^{\circ}$ at $h \nu=20 \mathrm{keV}$. The optical pathway is schematically shown in Fig. 1. The XFEL beam enters the SDU from the left side and is reflected by the first mirror (S1) into the direction of the beam splitter (BS). The green part of the beam is reflected into the upper delay path while the orange part passes the sharp edge of BS into the lower delay path. A path length difference and consequently a temporal delay of one beam path with respect to the other can be introduced by moving the mirrors $D_{1} / D_{2}$ or $U_{1} / U_{2}$ along the split beam directions. After the orange beam has passed the lower delay line it is reflected by the recombination mirror (RC) into the direction of the last mirror (S8). The green beam passes the sharp edge of the recombination mirror unaffected. The last mirror (S8) reflects both beams into their original direction towards the experiment. The beam shape of both arms is rotated by $180^{\circ}$ due to the odd number of reflections. For pump/probe experiments both beams have to be overlapped which can be achieved by slightly rotating the recombination mirror, RC. After passing the SDU the intense central parts, which are contiguous in the original beam, are distant due to the rotation of both partial beams by $180^{\circ}$. For some experiments it is desirable to overlap these parts at an angle $\phi$ as small as possible. In particular, for a measurement of the temporal coherence the width of the interference fringes depends on the overlap

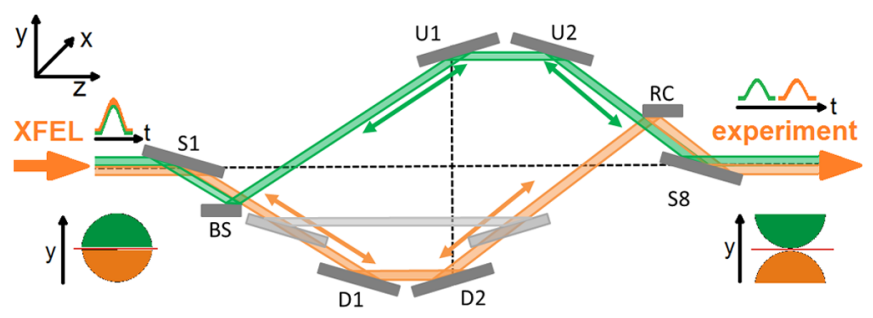

FIG. 1. The optical layout of the SDU for the HED experiment at the SASE2 undulator of the European XFEL. angle of both partial beams [compare Eq. (6)]. In order to resolve these fringes on a detector with a sufficient spatial resolution the angle $\phi$ has to be kept small. For this purpose the vertical position $y$ of $\mathrm{RC}$ is adjustable. When RC is moved vertically closer to the optical axis the less intense parts of both beams are cut at the edge of RC and the more intense central parts of the original beam move closer to each other. Consequently, for the partial beam in the lower branch the size of the footprint on the recombination mirror depends on the position of RC. If RC is aligned such that a vertical fraction $d_{y}$ of the beam can pass the footprint on the mirror will be $l=d_{y} / \sin \theta$. Though BS will reflect the full upper partial beam the less intense part also of this beam will be cut by the edge of RC. Thus, the effective footprint on BS that is relevant for the simulations is the same as for RC. The Bragg mirrors reflect at a given angle only a certain photon energy, and therefore they have to be rotated for optimal reflection when tuning the FEL. In order to transmit a wide photon energy range three different multilayer systems are coated on the mirror substrates beside each other. For photon energies between $5 \mathrm{keV}<h \nu<10 \mathrm{keV} \mathrm{Ni} / \mathrm{B}_{4} \mathrm{C}$ coatings with a multilayer period of $d=4 \mathrm{~nm}$ will be applied on all mirrors except for BS and RC. These two mirrors have to be irradiated at Bragg angles twice as large as the other mirrors. Here $\mathrm{W} / \mathrm{B}_{4} \mathrm{C}$ coatings with a period of $d=1.96 \mathrm{~nm}$ are used, because $\mathrm{Ni} / \mathrm{B}_{4} \mathrm{C}$ coatings with a period below $d=3 \mathrm{~nm}$ cannot be manufactured due to interdiffusion processes between the layers. For photon energies above $h \nu=10 \mathrm{keV} \mathrm{Mo/} / \mathrm{B}_{4} \mathrm{C}$ multilayers with a period of $d=$ $3.2 \mathrm{~nm}$ are used. Since for multilayers the grazing angle depends on the wavelength, the mirrors and consequently the whole beam path have to be adjusted for different photon energies. Hence, the maximum possible delay will vary as a function of the photon energy between $\Delta t= \pm 2.5 \mathrm{ps}$ at $h \nu=20 \mathrm{keV}$ and up to $\Delta t= \pm 23 \mathrm{ps}$ at $h \nu=5 \mathrm{keV}$. The selection of a specific mirror coating is facilitated by moving the whole setup horizontally in the $x$ direction perpendicular to the XFEL beam. In addition, two color experiments will be possible employing the fundamental and the third harmonic radiation. Therefore, a third coating is applied on the mirrors $\mathrm{S} 1$ and $\mathrm{S} 8$ because these two mirrors will have to reflect both photon energies at the same Bragg angle. Since this is not possible with a single multilayer coating, a novel two-color multilayer Bragg mirror has been developed [29]. This mirror consists of a Si substrate that is coated with two different types of multilayer systems, $n=120 \mathrm{Mo} / \mathrm{B}_{4} \mathrm{C}$ layers with a periodicity of $d=3.2 \mathrm{~nm}$ directly on the substrate and $n=4 \mathrm{Ni} / \mathrm{B}_{4} \mathrm{C}$ layers with a periodicity of $d=11.85 \mathrm{~nm}$ on top. Fundamental radiation with photon energies between 5 and $6.7 \mathrm{keV}$ is reflected by the $\mathrm{Ni} / \mathrm{B}_{4} \mathrm{C}$ multilayer system while the third harmonic $(15 \mathrm{keV}<$ $h \nu<20 \mathrm{keV}$ ) traverses this system and is reflected by the $\mathrm{Mo} / \mathrm{B}_{4} \mathrm{C}$ multilayers.

An important issue for all optics exposed to the intense $\mathrm{X}$-ray beam of the European XFEL is the risk of single shot 
damage. The damage thresholds found by theoretical calculations [30] as well as experimental results [31] for different types of multilayers are higher by more than 2 orders of magnitude than in the present case with expected fluences below $0.3 \mathrm{~mJ} / \mathrm{cm}^{2}$.

\section{METROLOGY RESULTS}

The quality of optical elements is one of the critical topics in the design of hard x-ray beam lines and instrumentation. These optics can be described by their deviation from ideal shape at different spatial frequencies. Usually one distinguishes between the figure error, the low spatial error part ranging from aperture length to $1 \mathrm{~mm}$ frequencies often described as slope error and the mid-spatial and high spatial roughness part from $1 \mathrm{~mm}$ to $1 \mu \mathrm{m}$ and from $1 \mu \mathrm{m}$ to some $10 \mathrm{~nm}$, respectively. While the slope error is contributing to aberration effects, the mid-spatial and high spatial roughness causes small and wide angle scatter. For the topics discussed in this paper particularly the knowledge of the mirror profiles is required in order to evaluate the impact of figure deviations on the wavefront of the XFEL pulse. For this purpose the mirrors were inspected by use of the nanometer optic component measuring machine (NOM) [32] at the BESSY-II metrology laboratory at HZB in Berlin. The NOM allows a precise measurement of such optics by use of slope measuring deflectometry with sub-nm precision [33] and a spatial resolution of $1.7 \mathrm{~mm}$ up to a length of $1200 \mathrm{~mm}$ [34]. Data acquired with the NOM provides an essential input for subsequent optimization technologies such as ion beam figuring and for the simulations presented in this paper.

Figure 2 exemplarily shows three of these mirror profiles measured along the center line along the $x=30 \mathrm{~mm}$ broad substrates. With a peak-to-valley (PV) error of only $\Delta h=$ $4 \mathrm{~nm}$ mirror 1 (green line) is well suited for an application in the SDU. In comparison the surface profile of mirror 2 (red, dashed line) shows a sine-like profile and a height

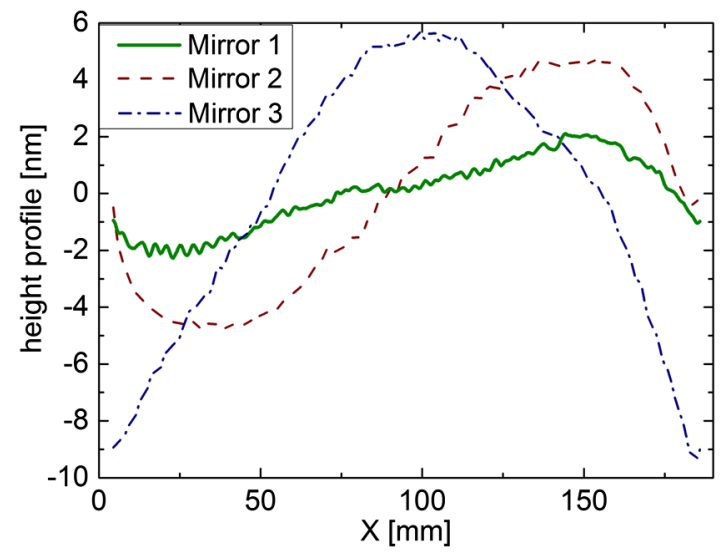

FIG. 2. The height profile of three mirrors: mirror 1 (green line) $\Delta h=4 \mathrm{~nm} \mathrm{PV}$; mirror 2 (red, dashed line) $\Delta h=9.5 \mathrm{~nm} \mathrm{PV}$; mirror 3 (blue, dash-dotted line) $\Delta h=15 \mathrm{~nm}$ PV. error accounting to $\Delta h=9.5 \mathrm{~nm}$. This mirror was used for the simulations presented in this paper. In the configuration that was simulated the footprint of the beam irradiates an $l=24 \mathrm{~mm}$ long area on the mirror. For the simulation of BS and RC the first $z=2 \mathrm{~mm}$ to $z=26 \mathrm{~mm}$ of mirror 2 and the last $z=166 \mathrm{~mm}$ to $z=190 \mathrm{~mm}$, respectively, have been used. The height error is $\Delta h=4.7 \mathrm{~nm}(\mathrm{PV})$ in both cases. For mirror 3 (blue, dash-dotted line) the peakto-valley error amounts to $\Delta h=15 \mathrm{~nm}$.

\section{METHODS}

The example of a measurement of the temporal coherence properties by means of the SDU descriptively shows the necessity of a high-accuracy wavefront propagation software. In the SRW package, a Fourier-optics based approach is used for the wavefront propagation simulation. The code has IGOR Pro and Python interfaces available, and it has already proven its utility for a large number of applications in UV, soft and hard x-ray spectral range [25]. The WPG framework improves high-level Python based access to the SRW functions, and includes HDF5 files support, interactive user interface and a set of routines specific for XFEL [26,27]. The software takes into account optics imperfections and allows modeling essential optical elements such as grazing incidence plane and focusing mirrors, gratings and compound refractive lenses. For the evaluation of the optical performance of the SDU mirrors a Python script that incorporates SRW and WPG library was written. This script uses as input a simulated XFEL pulse that is obtained from performed time-dependent simulations applying the SASE code FAST [28].

\section{A. Coherence of optical fields}

Coherence is one of the most outstanding features of FEL radiation. It can be well described in terms of statistical optics $[35,36]$. The spatiotemporal coherence can be measured by means of an SDU. For this purpose the partial beams are overlapped on an imaging detector and the interference fringes are recorded for different delays $\tau$ between the two beams. The complex degree of the mutual coherence $\gamma_{12}(\tau)$ is given as the normalized mutual coherence function $\Gamma\left(\mathbf{r}_{1}, \mathbf{r}_{2}, \tau\right)$ that is defined by the correlations of an electromagnetic wave field $\mathbf{E}(\mathbf{r}, t) . \Gamma\left(\mathbf{r}_{1}, \mathbf{r}_{2}, \tau\right)$ is described by the correlation function of the wave field $\mathbf{E}(\mathbf{r}, t)$ at two positions $\mathbf{r}_{1}$ and $\mathbf{r}_{2}$ at two different times $t$ and $(t+\tau)[35]$ :

$$
\Gamma\left(\mathbf{r}_{1}, \mathbf{r}_{2}, \tau\right)=\left\langle\mathbf{E}\left(\mathbf{r}_{1}, t\right) \mathbf{E}^{*}\left(\mathbf{r}_{2}, t+\tau\right)\right\rangle .
$$

A direct and easily realizable way to gain information about the absolute value of the normalized correlation function

$$
\left|\gamma_{12}(\tau)\right|=\left|\frac{\Gamma\left(\mathbf{r}_{1}, \mathbf{r}_{2}, \tau\right)}{\sqrt{\Gamma\left(\mathbf{r}_{1}, \mathbf{r}_{1}, 0\right) \Gamma\left(\mathbf{r}_{2}, \mathbf{r}_{2}, 0\right)}}\right|
$$


is calculating the visibility $V$ of the interference fringes of two interfering partial beams. This visibility $V$ is given by the maximum and adjacent minimum intensity of the interference fringes

$$
V=\frac{I_{\max }-I_{\min }}{I_{\max }+I_{\min }}=\left|\gamma_{12}(\tau)\right|\left\{2 \sqrt{I_{1} I_{2}} /\left(I_{1}+I_{2}\right)\right\},
$$

where $I_{1}$ and $I_{2}$ denote intensities of the interfering partial beams and $I_{\max }$ and $I_{\min }$ are the maximum and minimum intensities of the interference fringes. The intensity on the detector at a position $y_{d}$ is given by

$$
\begin{aligned}
I\left(y_{d}, \tau\right)= & {\left[I_{1}\left(y_{d}\right)+I_{2}\left(y_{d}\right)\right] } \\
& +2 \sqrt{I_{1}\left(y_{d}\right) I_{2}\left(y_{d}\right)}\left|\gamma_{12}(\tau)\right| \cos \left[k \phi y_{d}+\alpha_{12}(\tau)\right],
\end{aligned}
$$

where $I_{1,2}\left(y_{d}\right)=\left\langle E_{1,2}^{2}\left(y_{d}, t\right)\right\rangle, k$ is the wave number and $\gamma_{12}(\tau)$ is the complex degree of coherence, and $\alpha_{12}(\tau)$ is the phase of the complex degree of coherence. The first term accounts for the intensities of the two partial beams. The second term introduces a modulation via the cosine function. Here $k \phi y_{d}$ accounts for the slightly different angle of incidence $|\phi|$ of the second beam that is necessary to overlap both beams. For a partially coherent light source the complex degree of coherence $\gamma_{12}(\tau)$ decreases with increasing time delay $\tau$. If the detector is arranged in a distance $z$ from the SDU and the beams are overlapped on the detector by $\Delta y_{d}$ the interferences will show a spatial period length

$$
l=\frac{\lambda \cdot z}{\Delta y_{d}}
$$

on the detector with $\phi=y_{d} / z$. The temporal coherence can be described by $\left|\gamma_{12}(\tau)\right|$ as a function of $\tau$ for fixed points $r_{1}$ and $r_{2}$. The coherence time $\tau_{c}$ is defined differently in the literature. It can be defined as the half width at half maximum [HWHM] or as the width at a value of $1 / \mathrm{e}$ of the maximum of a Gaussian function fitted to $\left|\gamma_{12}(\tau)\right|$. A more general definition for an arbitrary function is given by the rms value [35]

$$
\tau_{c, r m s}=\int_{-\infty}^{\infty}\left|\gamma_{12}(\tau)\right|^{2} d \tau
$$

For a Gaussian function this yields $\tau_{c, r m s} \approx 0.85 \tau_{c}$. However, a calculation of the visibility is only suitable if solely time-dependent two-beam interference is the origin of the fringes. Additional well-defined fringes that may occur for instance from diffraction at the edge of the beam splitter can still be tolerated in most cases. In contrast, wavefront distortions caused by imperfect mirrors are capable of provoking fully modulated arbitrary disturbances of the intensity distribution on the detector. In this case an accurate determination of the temporal coherence properties by calculating the visibility of the fringes is difficult. Therefore, a different approach has to be applied. First, the interference pattern that is measured on the detector is Fourier transformed. According to $[9,37]$ the Fourier transform is connected to the complex coherence function $\gamma_{12}(\tau)$ via

$$
\begin{aligned}
\tilde{I}(f)= & 2 \tilde{I}_{1}(f) *\left\{\delta(f)+\frac{1}{2}\left|\gamma_{12}(\tau)\right|\left[\delta\left(f+f_{s}\right) e^{-i \alpha_{12}(\tau)}\right.\right. \\
& \left.\left.+\delta\left(f-f_{s}\right) e^{i \alpha_{12}(\tau)}\right]\right\},
\end{aligned}
$$

where $\tilde{I}(f)$ denotes the Fourier transform of $I\left(y_{d}\right), \delta$ is the dirac delta function, $*$ is the convolution operator, and $f_{s}$ is the spatial frequency of the interference fringes. The signal originating from time-dependent two-beam interference then occurs at this well-defined spatial frequency determined by $\Delta y_{d} / \lambda z$, see Eqs. (5) and (6). The signal decreases for increasing temporal delays, while the signal caused by wavefront distortions is spread arbitrarily and stays constant for large delays. By normalizing the signal of the Fourier transform $\tilde{I}(f)$ for different delays $\tau$ to the signal at zero delay the coherence time $\tau_{c}$ can be determined.

\section{B. Computational methods}

This section briefly summarizes the description that is presented in Ref. [25] about the Fourier optics approach applied in SRW software.

The complex electric field $\mathbf{E}(\mathbf{r}, t)$ in the time domain is connected to the field $\mathbf{E}(\mathbf{r}, \omega)$ in the frequency domain via the Fourier transform

$$
\mathbf{E}(\mathbf{r}, \omega)=\int_{-\infty}^{\infty} \mathbf{E}(\mathbf{r}, t) e^{i \omega t} d t
$$

and

$$
\mathbf{E}(\mathbf{r}, t)=\frac{1}{2 \pi} \int_{-\infty}^{\infty} \mathbf{E}(\mathbf{r}, \omega) e^{-i \omega t} d \omega
$$

For small emission and observation angles the propagation of the transverse components in the frequency domain from a point $\mathbf{r}_{1}$ towards a point $\mathbf{r}_{2}$ can be described in terms of the Huygens-Fresnel principle by an integration over the plane $\Sigma_{1}$ perpendicular to the $z$ axis,

$$
\mathbf{E}_{\perp}\left(\mathbf{r}_{\mathbf{2}}, \omega\right) \approx \frac{-i \omega}{2 \pi c} \iint \mathbf{E}_{\perp}\left(\mathbf{r}_{\mathbf{1}}, \omega\right) \frac{e^{i \omega\left|\mathbf{r}_{2}-\mathbf{r}_{1}\right| / c}}{\left|\mathbf{r}_{\mathbf{2}}-\mathbf{r}_{\mathbf{1}}\right|} d \Sigma_{1} .
$$

If the plane $\Sigma_{1}$ is perpendicular to the $z$ axis, and $\mathbf{r}_{2}$ belongs to another plane $\Sigma_{2}$ located at a distance $\Delta z$ from $\Sigma_{1}$, then Eq. (10) is a convolution integral with $d \Sigma=d x_{1} d y_{1}$ and $\left|\mathbf{r}_{2}-\mathbf{r}_{\mathbf{1}}\right|=\left[\Delta z^{2}+\left(x_{2}-x_{1}\right)^{2}+\left(y_{2}-y_{1}\right)^{2}\right]^{1 / 2}$. This can be 
numerically solved by means of a $2 \mathrm{D}$ fast Fourier transformation (FFT).

To increase the numerical efficiency of the calculation without a loss of accuracy (in the small-angle approximation) the square root can be approximated by the first terms of its Taylor series decomposition in the exponent argument and by $\delta z$ outside the exponent. This allows for applying an analytical treatment of the quadratic phase term, resulting in a considerable reduction of the electric field sampling rate required for the numerical FFT-based processing. Furthermore, a set of 2D FFTs for different frequency (photon energy) values can be performed "in place" for one flat array of the complex electric field data sampled vs the photon energy, horizontal and vertical position. Such an efficient algorithm, which was implemented in SRW code, allows for performing simulation of propagation of a FEL pulse described by gigabytes of the electric field data through a complicated optical system within a time interval ranging from several minutes to half an hour even on one CPU.

\section{TIME-DEPENDENT WAVEFRONT PROPAGATION}

The wavefront propagation simulations were carried out for a photon energy of $h \nu=4.96 \mathrm{keV}$. As starting conditions the output of the FEL simulation code FAST applied to the operation conditions of the XFEL accelerator at $E_{\text {Bunch }}=14 \mathrm{GeV}$ and the properties of the SASE2 undulator were used. The charge of the electron bunches in this simulation was $Q=100 \mathrm{pC}$. In this way, saturated SASE pulses with an angular divergence of $\Theta=3.87 \mu \mathrm{rad}$ (FWHM) are generated. After leaving the undulator the beam propagates towards the SDU which will be located $846 \mathrm{~m}$ behind the undulator. Here the beam diameter is $d=3.27 \mathrm{~mm}$ (FWHM). At BS the beam is split into two partial beams that are delayed with respect to each other. The vertical position $y$ of $\mathrm{RC}$ is aligned to accept $d_{y}=$ $1.5 \mathrm{~mm}$ of the partial beam from the lower delay branch (orange in Fig. 1). Thus, also the other partial beam is cut by the edge of $\mathrm{RC}$ such that $d_{y}=1.5 \mathrm{~mm}$ will pass. Consequently only the first $l=d_{y} / \sin \left(3.6^{\circ}\right)=24 \mathrm{~mm}$ of the mirror profile are irradiated. The peak-to-valley height error within this footprint amounts to $\Delta h=4.7 \mathrm{~nm}$. The two partial beams then propagate $130 \mathrm{~m}$ before they irradiate the detector or interact with a sample in the experimental area. Since BS and RC reflect the beam at a Bragg angle that is twice as large compared to the delay mirrors the contribution of BS and $\mathrm{RC}$ to the total wavefront distortion will be dominant according to Eq. (1). Thus, for this study only BS and RC are taken into account. In this case the metrology data of mirror 2 was used. Figure 3(a) shows for zero delay the temporal shape of the FEL pulse $(\Delta t \approx 10 \mathrm{fs})$ with its typical spikes that originate from the statistical behavior of the SASE process [38]. Applying a splitting ratio of 1:1 Figs. 3(b) and 3(c) show
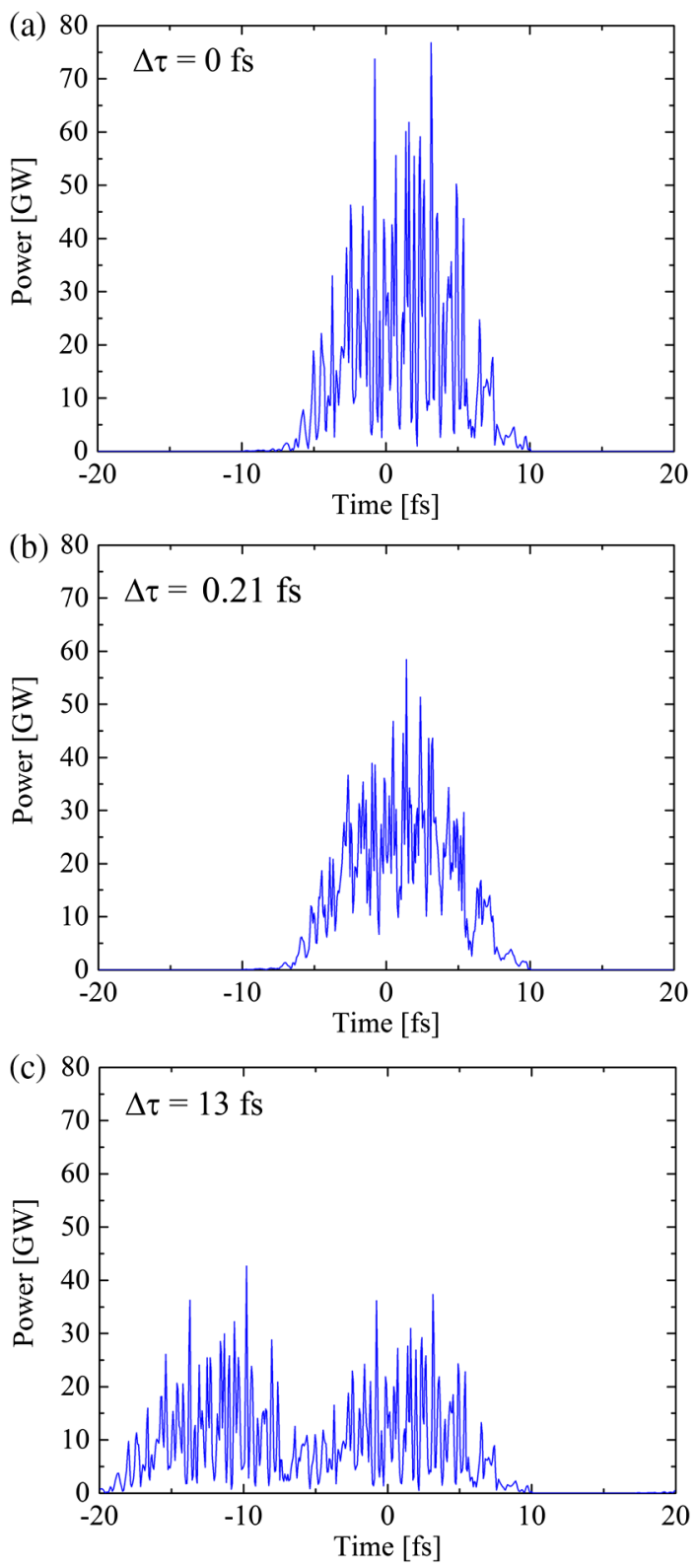

FIG. 3. The temporal shape of the FEL pulse at $h \nu=4.96 \mathrm{keV}$ for (a) zero delay, (b) $\tau=0.21$ fs and (c) $\tau=13$ fs where the split pulses are clearly separated.

the temporal profile of the FEL pulses behind the SDU for two delays $\Delta \tau=0.21 \mathrm{fs}$ and $\Delta \tau=13 \mathrm{fs}$. In the latter case the split pulses are clearly separated. Figure 4 shows the spectrum of the SASE pulse used for the simulation with a spectral width of $\Delta E \approx 5 \mathrm{eV}$. Also in the spectrum the typical spikes of SASE pulses are apparent. Figure 5(a) shows the beam profile of the two separated beams for ideal flat mirrors in the SDU. As mentioned before the wavefront distortion caused by the offset beam line mirror is significantly smaller than the distortion caused by the mirrors in the SDU. Therefore it is not taken into account for the simulation. Here only fringes from diffraction at the edges of the beam splitter (BS) and recombination mirror (RC) 


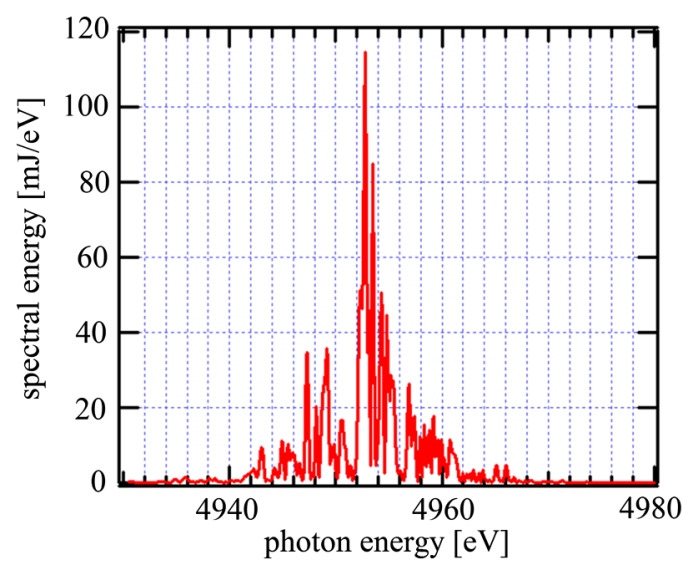

FIG. 4. The spectrum of the FEL pulse that was used for the wavefront propagation simulation.

are apparent. In comparison, Fig. 5(b) shows the same case for mirrors with a peak-to-valley error of up to $\Delta h=$ $4.7 \mathrm{~nm}$ within the footprint that is irradiated by the beam. For this simulation the profile of mirror 2 as shown in Fig. 2 was used. It is evident that the distortions of the wavefront introduced by the mirror cause considerable perturbations of the fringe pattern.

For a measurement of the temporal coherence the partial beams have to be overlapped in order to generate an interference pattern that can be evaluated for different delays. Figure 6 shows this configuration for zero delay, again for ideal flat mirrors (a) and for mirrors with $\Delta h=$ $4.7 \mathrm{~nm}$ (b). In the central area they are overlapping each other by $\Delta y_{d}=0.75 \mathrm{~mm}$ at an angle of $\phi=5.8 \mu \mathrm{rad}$ which corresponds to $23 \%$ of the beam profile in the vertical direction as it is incident on the detector. For a better visualization of the modulation a vertical cut through

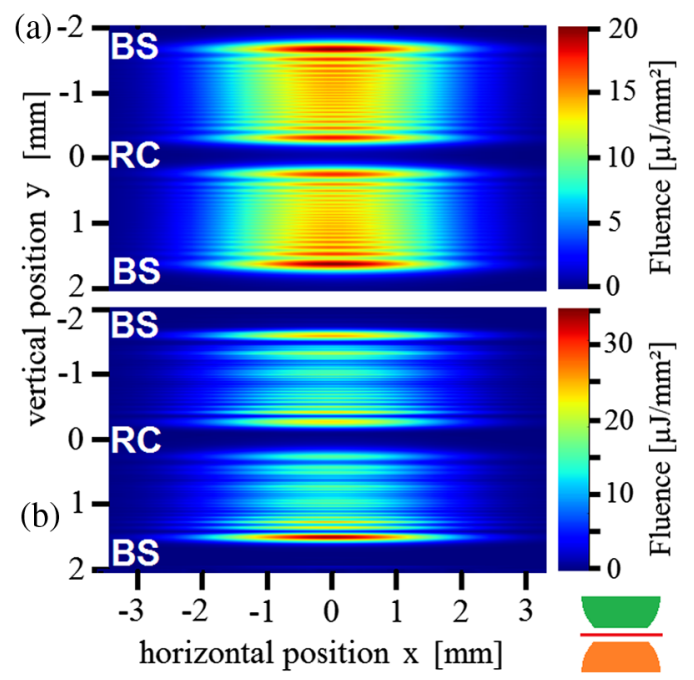

FIG. 5. The XFEL beam on the imaging detector at $z=976 \mathrm{~m}$ split by the SDU containing ideal flat mirrors (a) and mirrors with the height profile of mirror 2 (b). the interference pattern that is shown in Fig. 6 at $x=0$ is depicted in Fig. 7(a). Due to the ideal mirror surfaces the modulation is perfectly symmetric. In the areas where the beams do not overlap interference fringes from diffraction at the edge of the beam splitter appear. At $\tau=0$ fs the interference pattern is almost fully modulated in the region where the beams overlap. The spatial frequency of the fringes amounts to $f=23$ periods $/ \mathrm{mm}$ as it is expected from Eq. (6). A visibility of $V=0.96$ is calculated using Eq. (4). When the delay $\tau$ between both beams is increased, the modulation of the interference fringes first decreases and for large delays eventually completely vanishes. Accordingly, for a delay of $\Delta \tau=0.21 \mathrm{fs}$ the visibility decreases to $V=0.41$, Fig. 7(b). In Fig. 7(c), where the delay between both beams has been increased to $\tau=13 \mathrm{fs}$ the fringes from time-dependent two-beam interference completely vanish and only fringes from diffraction at the mirror edges are apparent.

For nonperfect mirrors the situation looks very different, as it is obvious from Fig. 8. For zero delay again strong modulations appear. The interference pattern, however, is no longer symmetric with respect to the beam profile [Fig. 8(a)]. A visibility of $V \approx 0.91$ can still be derived. Further a decrease of the modulation depth can clearly be noticed for larger delays of 0.21 and 13 fs [Figs. 8(b) and 8(c)], as expected. However, considerable perturbations of the interference pattern by the wavefront distortions impede a direct evaluation of the visibility via Eq. (4). The highfrequency fringes which result from time-dependent twobeam interference appear at a fixed spatial frequency on the imaging detector. Therefore, their contribution to the entire pattern can be separated by means of a FFT [7,9]. Figure 9 shows the FT of the interference patterns for both cases, ideal mirrors Fig. 9(a) and nonperfect mirrors Fig. 9(b).

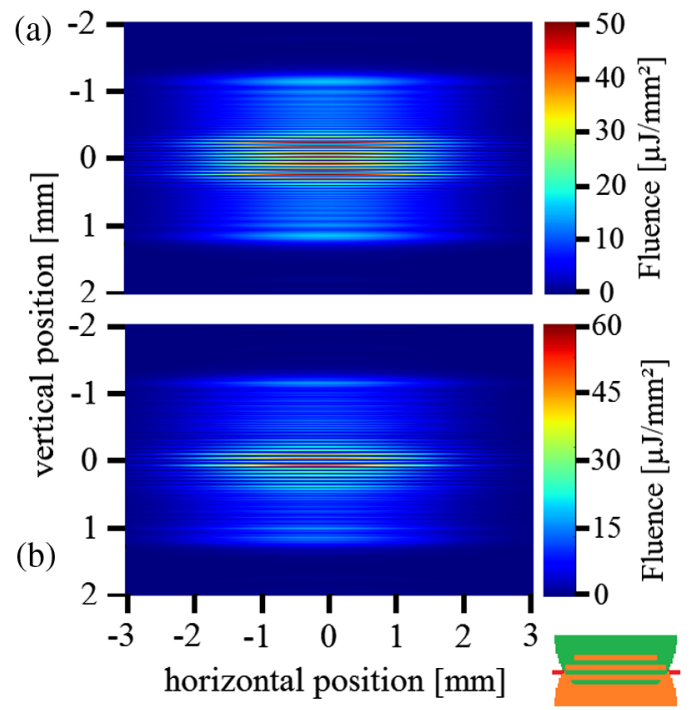

FIG. 6. Both half beams overlapping each other by $\Delta y_{d}=$ $0.75 \mathrm{~mm}$ for ideal flat mirrors (a) and for mirrors with $\Delta h=$ $4.7 \mathrm{~nm}$ within the footprint of the beam (b). 


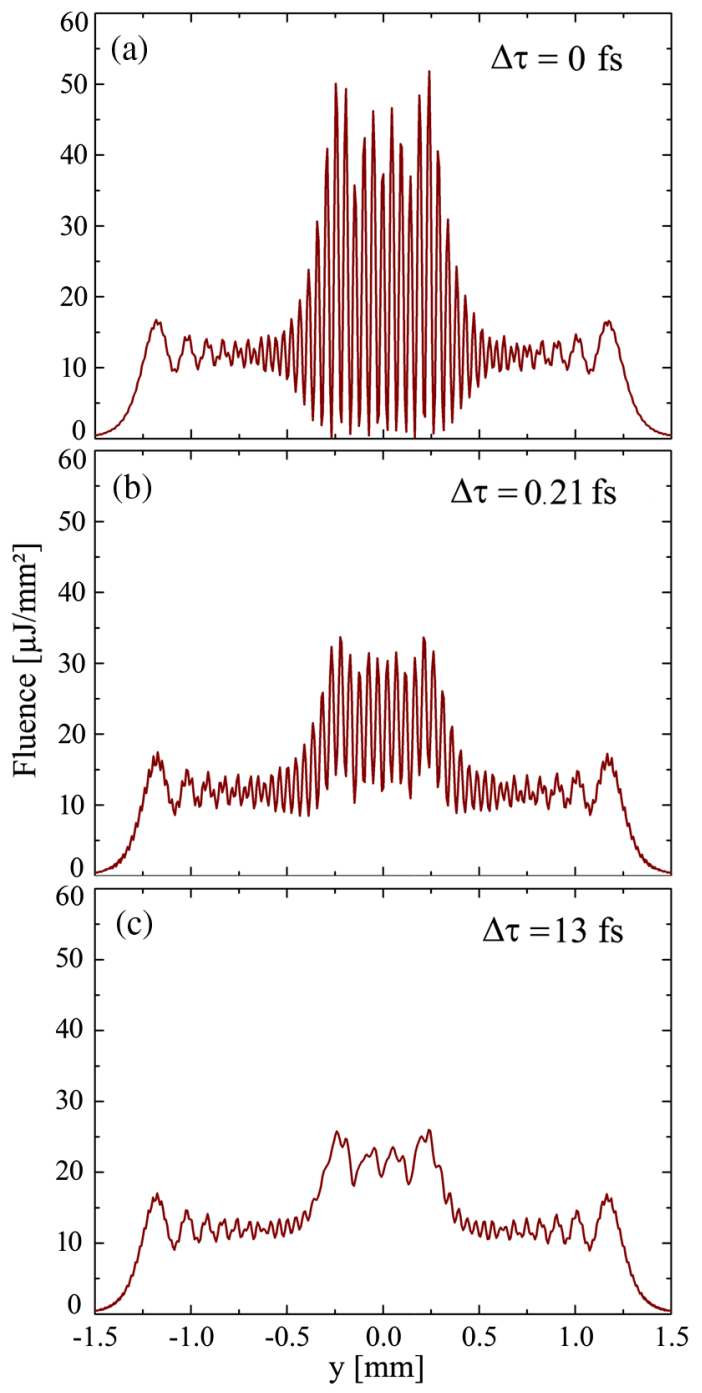

FIG. 7. Vertical cuts through the interference patterns for ideal flat mirrors for zero delay (a) for $\tau=0.21 \mathrm{fs}$ (b) and for $\tau=13$ fs (c).

It is obvious from Fig. 9(a) that the fringes caused by diffraction at the edges of the BS and RC contribute to the FT signal mainly at low spatial frequencies below 10 periods $/ \mathrm{mm}$. The two-beam interference maximum appears at a spatial frequency of $f=23.3$ periods $/ \mathrm{mm}$ as it is expected according to Eq. (6) from the geometrical parameters, with an overlap of $\Delta y_{d}=0.75 \mathrm{~mm}$ at an angle of $\phi=5.8 \mu \mathrm{rad}$, compare Eq. (6). The FT signal is normalized to this maximum at zero delay (red line). When the delay is set to $\tau=0.21$ fs (green line) the FT signal for ideal mirrors decreases to $V=0.43$ which is in a good agreement with the evaluation of the visibility via Eq. (4). For a delay of $\tau=13$ fs (blue line) the visibility is still $V=0.03$. Figure 9(b) shows the FT of the interference pattern for the SDU equipped with real, nonperfect mirrors. Due to the significant wavefront distortions the peaks are not perfectly symmetric. They are shifted somewhat to
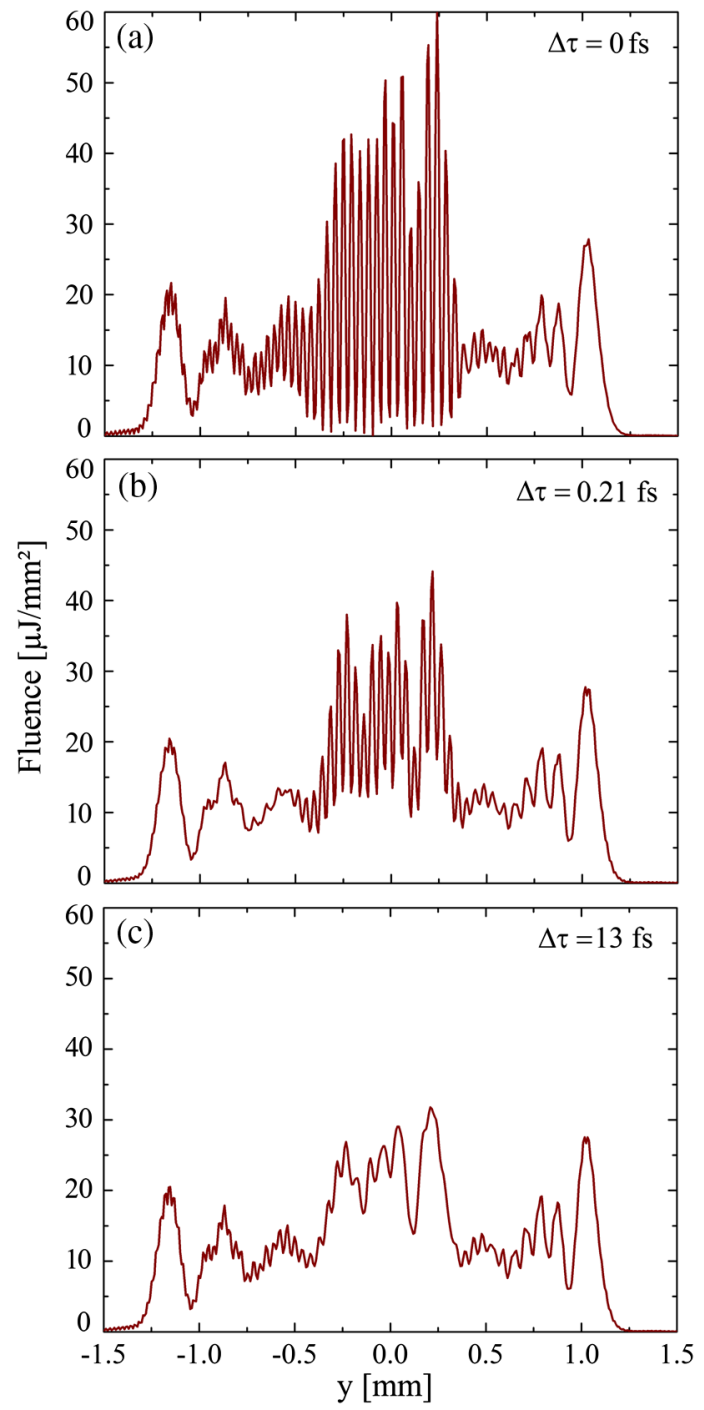

FIG. 8. Vertical cuts through the interference patterns for nonperfect mirrors for zero delay (a) for $\tau=0.21 \mathrm{fs}$ (b) and for $\tau=13$ fs (c).

higher spatial frequencies with a maximum at $f=25.1$ periods $/ \mathrm{mm}$. This higher modulation frequency can be explained by an additional angle $\phi_{\text {profile }}$ that is introduced by the height variation of $\Delta h=4.7 \mathrm{~nm}$ within the $24 \mathrm{~mm}$ footprint of the beam. This angle is then given as $\phi_{\text {profile }}=2 \Delta h / 24 \mathrm{~mm}=0.4 \mu \mathrm{rad}$ which corresponds to $7 \%$ of the overlap angle $\phi$ of the partial beams and would shift the spatial frequency of the interference fringes from $f_{s}=23.3 \mathrm{~nm}$ to $f_{s}=24.9 \mathrm{~nm}$. It is obvious that the peaks are not perfectly symmetric. Thus, for the evaluation of the temporal coherence it is not possible to just use the values of the maxima, instead the peak is integrated within the area marked by red lines in Fig. 9(b).

The result of a simulated measurement of the temporal coherence of the hard X-ray pulses is shown in Fig. 10 for both, ideal mirrors indicated by red dots and nonperfect mirrors indicated by blue triangles. In general, the results 

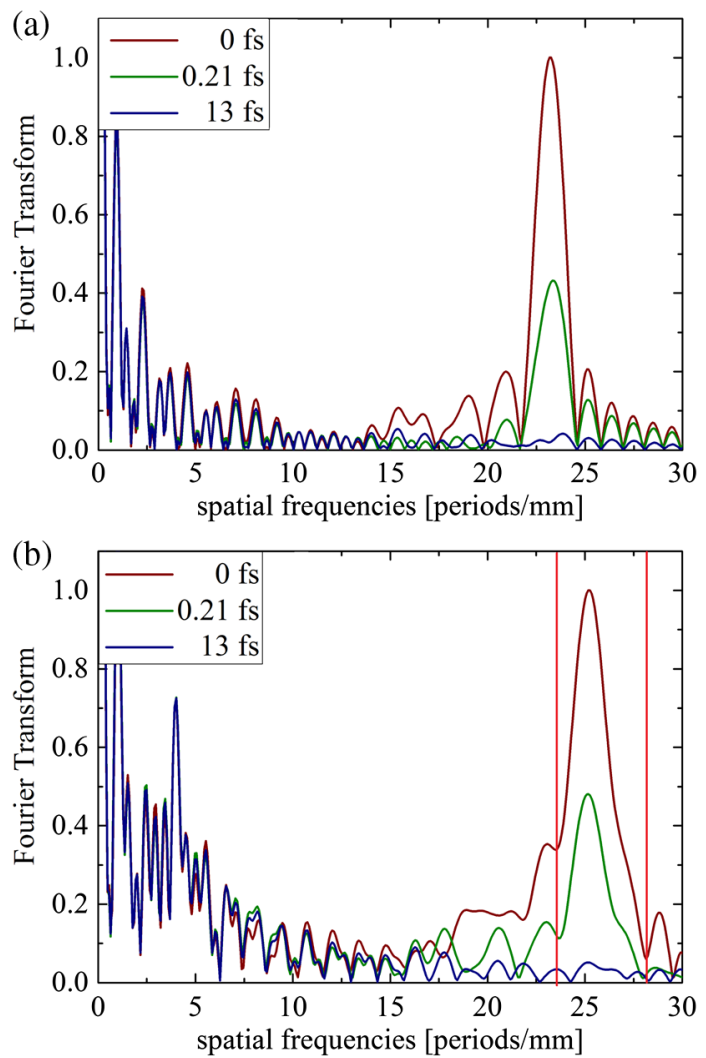

FIG. 9. Normalized FT for ideal mirrors (a) and for nonperfect mirrors (b). The red vertical lines in Fig. (b) mark the area that is integrated in order to evaluate the temporal coherence.

show the typical characteristics of the temporal coherence properties of SASE pulses. A narrow peak that can be approximated with a Gaussian function assuming a constant background of $V=0.2$ appears on short time scales

- ideal mirror surface

$\checkmark$ mirror 2

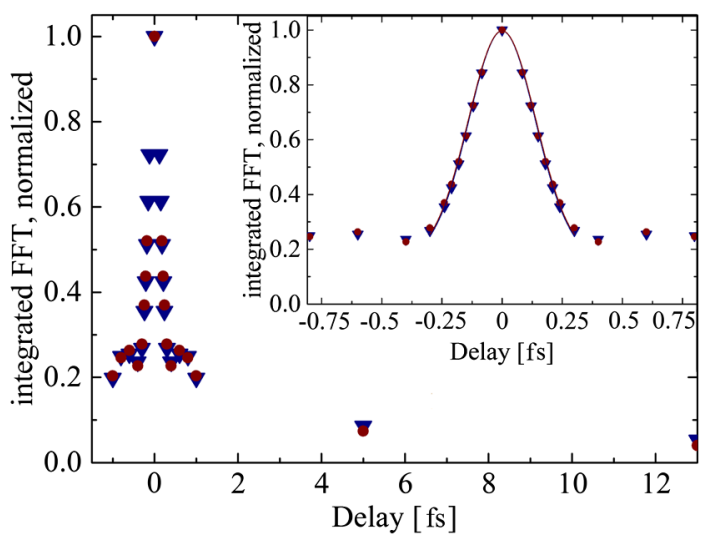

FIG. 10. A measurement of the temporal coherence properties of XFEL pulses for ideal mirrors (red dots) and real, nonperfect mirrors (blue triangles). The inset shows the magnified central peak with fitted Gaussian functions yielding a coherence time of $\tau_{c}=0.191 \mathrm{fs}$ (HWHM) for perfect mirrors (red) $\tau_{c, \mathrm{HWHM}}=$ $0.187 \mathrm{fs}$ (HWHM) for real, nonperfect mirrors (blue). of -0.3 fs $\leq \tau \leq 0.3$ fs. This can be explained by the coherence time of a single mode of the SASE pulse, which in this simulation amounts to $\tau_{c}=0.243$ fs. At $\tau=0.6$ fs there appears a secondary peak which can be explained by a phase-locked interference of subsequent temporal modes within the pulse. This behavior has experimentally been observed in similar measurements in the XUV spectral regime at FLASH at $h \nu=52 \mathrm{eV} \mathrm{[6].} \mathrm{In} \mathrm{other} \mathrm{experiments}$ at FLASH this secondary peak did not appear at a fixed position, but a gradual decay of the visibility on a longer time scale has been reported in all cases [7-9].

It is obvious from Fig. 10 that for long delays even at $\tau=13 \mathrm{fs}$ the measurement is disturbed by the interferences that are caused by wavefront distortions and by diffraction. At the spatial frequencies in question the contribution of the perturbation fringes still yields a visibility of $V=0.06$. In comparison, for perfect mirrors the fringes caused by diffraction at the edges of the beam splitter and recombination mirror yield a visibility of only $V=0.03$. It should be noted that the peaks can also shift to lower spatial frequencies when a defocusing mirror profile occurs. In that the contribution from the background will increase, resulting in a seemingly increase of the visibility at large delays. However, this shift can be compensated by increasing the overlap $y_{d}$ which results in a higher spatial frequency of the interference fringes according to Eq. (6). The main goal of such an experiment is to find a reliable value for the coherence time. The inset of Fig. 10 shows the magnified central peak with approximated Gaussian functions which yield coherence times of $\tau_{c}=0.191 \mathrm{fs}$ (HWHM) for perfect mirrors (red) and $\tau_{c}=0.187 \mathrm{fs}$ (HWHM) for real, nonperfect mirrors (blue), respectively. Accordingly, the systematical error caused by the wavefront distortions amounts to only $\Delta \tau_{c}=4 \times 10^{-3}$ fs (HWHM) which corresponds to only $2.1 \%$. In comparison, the uncertainty of the coherence time that is caused by shot-to-shot fluctuations of the SASE pulses is typically much higher. For instance, in the measurements presented in Ref. [8] the statistical uncertainty of the coherence time was typically $8 \%$ to $17 \%$ for wavelengths between $\lambda=8 \mathrm{~nm}$ $(h \nu=155 \mathrm{eV})$ and $\lambda=32 \mathrm{~nm}(h \nu=39 \mathrm{eV})$. Hence, the novel hard X-ray SDU will enable a reliable experimental evaluation of the temporal coherence properties of the hard $\mathrm{x}$-ray pulses generated by the European XFEL, even with wavefront distorting mirrors with a peak-to-valley height difference of $\Delta h=4.7 \mathrm{~nm}$ within the area that is irradiated by the footprint of the XFEL beam. It should be noted that the simulated interference patterns represent single pulses of the European XFEL. Angular vibrations of the mirrors on the order of 20 to 100 nrad would wash out the high spatial frequency components of the interferences on the detector for subsequent pulses. However, the European XFEL with it superconducting accelerator is capable of delivering pulse trains of up to 2700 pulses within a $600 \mu \mathrm{s}$ burst. Within this very short time the vibrating motion of 
the mirrors is too low to affect the experiment. Thus, it will be possible to integrate over the hard x-ray pulse train and determine their average temporal coherence properties.

\section{CONCLUSION}

In this paper a detailed simulation of the influence of nonperfect mirrors on a measurement of the temporal coherence properties of hard x-ray pulses at $h \nu=5 \mathrm{keV}$ generated by the European XFEL is presented. The timedependent wavefront propagation simulation has been performed using SRW software [25,26]. In this regard the software has proven to be a powerful tool for this kind of simulations. Wavefront distortions caused by nonperfect mirrors lead to considerable perturbations of the interference patterns. However, the relevant fringes resulting from time-dependent two-beam interference can be filtered and evaluated by means of a Fourier transformation based technique. In this way coherence times of $\tau_{c}=0.187 \mathrm{fs}$ (HWHM) and $\tau_{c}=0.191 \mathrm{fs}(\mathrm{HWHM})$ for found for real nonperfect and for ideal mirrors, respectively. The systematic error of the measurement of $2.1 \%$ can be tolerated since the shot-to-shot fluctuations of the temporal coherence of the SASE pulses are considerably larger.

\section{ACKNOWLEDGEMENT}

The authors gratefully acknowledge the financial support by the Bundesministerium für Bildung und Forschung via Grants No. 05K10PM2 and No. 05K13PM1 within the research program FSP 302 "Freie-Elektronen-Laser: Kondensierte Materie unter extremen Bedingungen." Part of the metrology work was funded by the European Metrology Research Project-EMRP-JRP SIB58 Angles within the EURAMET program of the European Union.

[1] P. Emma, R. Akre, J. Arthur, R. Bionta, C. Bostedt, J. Bozek, A. Brachmann, P. Bucksbaum, R. Coffee, F.-J. Decker et al., Nat. Photonics 4, 641 (2010).

[2] H. Tanaka, M. Yabashi et al., Nat. Photonics 6, 540 (2012).

[3] M. Altarelli et al., The European XFEL Technical Design Report, Desy, 2006.

[4] H. Chapman, A. Barty, M. Bogan, S. Boutet, M. Frank, S. Hau-Riege, S. Marchesini, B. Woods, S. Bajt, and W. Benner, Nat. Phys. 2, 839 (2006)

[5] C. M. Gúnther, B. Pfau, R. Mitzner, B. Siemer, S. Roling, H. Zacharias, O. Kutz, I. Rudolph, D. Schondelmaier, R. Treusch, and S. Eisebitt, Nat. Photonics 5, 99 (2011).

[6] R. Mitzner, B. Siemer, M. Neeb, T. Noll, F. Siewert, S. Roling, M. Rutkowski, A. A. Sorokin, M. Richter, P. Juranic, K. Tiedtke, J. Feldhaus, W. Eberhardt, and H. Zacharias, Opt. Express 16, 19909 (2008).

[7] W. F. Schlotter, F. Sorgenfrei, T. Beeck, M. Beye, S. Gieschen, H. Meyer, M. Nagasono, A. Föhlisch, and W. Wurth, Opt. Lett. 35, 372 (2010).
[8] S. Roling, B. Siemer, M. Wöstmann, H. Zacharias, R. Mitzner, A. Singer, K. Tiedtke, and I. A. Vartanyants, Phys. Rev. ST Accel. Beams 14, 080701 (2011).

[9] A. Singer, F. Sorgenfrei, A. P. Manusco, N. Gerasimova, O. M. Yefanov, J. Golden, T. Gorniak, T. Senkbeil, A. Sakdinawat, Y. Liu, D. Attwood, A. Dziarzhytski, D. D. Mai, R. Treusch, E. Weckert, T. Salditt, A. Rosenhahn, W. Wurth, and I. A. Vartanyants, Opt. Express 20, 17480 (2012).

[10] R. Mitzner, A. A. Sorokin, B. Siemer, S. Roling, M. Rutkowski, H. Zacharias, M. Neeb, T. Noll, F. Siewert, W. Eberhardt, M. Richter, P. Juranic, K. Tiedtke, and J. Feldhaus, Phys. Rev. A 80, 025402 (2009).

[11] M. Wöstmann, R. Mitzner, T. Noll, S. Roling, B. Siemer, F. Siewert, F. Wahlert, and H. Zacharias, J. Phys. B 46, 164005 (2013).

[12] F. Sorgenfrei, W. F. Schlotter, T. Beeck, M. Nagasono, S. Gieschen, H. Meyer, A. Föhlisch, M. Beyer, and W. Wurth, Rev. Sci. Instrum. 81, 043107 (2010).

[13] B. F. Murphy, J.-C. Castagna, J. D. Bozek, and N. Berrah, Proc. SPIE Int. Soc. Opt. Eng. 8504, 850409 (2012).

[14] R. Sobierajski, R. A. Loch, R. W. E. van de Kruijs, E. Louis, G. von Blanckenhagen, E. M. Gullikson, F. Siewert, A. Wawro, and F. Bijkerk, Rev. Sci. Instrum. 81, 043107 (2010).

[15] V. Hilbert et al., Appl. Phys. Lett. 105, 101102 (2014).

[16] W. Roseker, H. Franz, H. Schulte-Schrepping, A. Ehnes, O. Leupold, F. Zontone, A. Robert, and G. Grübel, Opt. Lett. 34, 1768 (2009).

[17] T. Osaka, T. Hirano, M. Yabashi, Y. Sano, K. Tono, Y. Inubushi, T. Sato, K. Ogawa, S. Matsuyama, T. Ishikawa, and K. Yamauchi, Proc. SPIE Int. Soc. Opt. Eng. 9210, 921009 (2014).

[18] M. Krikunova, M. Adolph, T. Gorkhover, D. Rupp, S. Schorb, C. Bostedt, S. Roling, B. Siemer, R. Mitzner, H. Zacharias, and T. Möller, J. Phys. B 45, 105101 (2012).

[19] U. Zastrau et al., Phys. Rev. Lett. 112, 105002 (2014).

[20] L. Samoylova, H. Sinn, F. Siewert, H. Mimura, K. Yamouchi, and Th. Tschentscher, Proc. SPIE Int. Soc. Opt. Eng. 7360, 73600E (2009).

[21] S. Rutishauser, L. Samoylova, J. Krzywinski, O. Bunk, J. Grünert, H. Sinn, M. Cammarata, D. Fritz, and C. David, Nat. Commun. 3, 947 (2012).

[22] G. Geloni, E. Saldin, L. Samoylova, E. Schneidmiller, H. Sinn, Th. Tschentscher, and M. Yurkov, New J. Phys. 12, 035021 (2010).

[23] S. Roling, L. Samoylova, B. Siemer, H. Sinn, F. Siewert, F. Wahlert, M. Wöstmann, and H. Zacharias, Proc. SPIE Int. Soc. Opt. Eng. 8504, 850407 (2012).

[24] M. Nakatsumi, K. Appel, G. Priebe, I. Thorpe, A. Pelka, B. Muller, and Th. Tschentscher, TDR: Scientific Instrument High Energy Density (HED) XFEL.EU TR-2014-001 (2014).

[25] O. Chubar, M. E. Couprie, M. Labat, G. Lambert, F. Polack, and O. Tcherbakoff, Nucl. Instrum. Methods Phys. Res., Sect. A 593, 30 (2008).

[26] L. Samoylova, A. Buzmakov, G. Geloni, O. Chubar, and H. Sinn, Proc. SPIE Int. Soc. Opt. Eng. 8141, 81410A-1 (2011). 
[27] https://github.com/samoylv/WPG.

[28] E. L. Saldin, E. A. Schneidmiller, and M. V. Yurkov, Nucl. Instrum. Methods Phys. Res., Sect. A 429, 233 (1999).

[29] S. Roling, S. Braun, P. Gawlitza, M. Wöstmann, E. Ziegler, and H. Zacharias, Opt. Lett. 39, 2782 (2014).

[30] R. A. Loch, R. Sobierajski, E. Louis, J. Bosgra, and F. Bijkerk, Opt. Express 20, 28200 (2012).

[31] E. Louis, A. R. Khorsand, R. Sobierajski, E. D. van Hattum, M. Jurek, D. Klinger, J. B. Pelka, L. Juha, J. Chalupsky, J. Cihelka, V. Hajkova, U. Jastrow, S. Toleikis, H. Wabnitz, K. Tiedke, J. Gaudin, E. M. Gullikson, and F. Bijkerk, Proc. SPIE Int. Soc. Opt. Eng. 7361, 73610I (2013).

[32] F. Siewert, J. Buchheim, T. Zeschke, G. Brenner, S. Kapitzki, and K. Tiedtke, Nucl. Instrum. Methods Phys. Res., Sect. A 635, S52 (2011).
[33] F. Siewert, J. Buchheim, S. Boutet, G. J. Williams, P. A. Montanez, J. Krzywinski, and R. Signorato, Opt. Express 20, 4525 (2012).

[34] F. Siewert, J. Buchheim, T. Höft, T. Zeschke, A. Schindler, and T. Arnold, Nucl. Instrum. Methods Phys. Res., Sect. A 710, 42 (2013).

[35] J. W. Goodman, Statistical Optics, 1st ed. (WileyInterscience, New York, 1985).

[36] L. Mandel and E. Wolf, Optical Coherence and Quantum Optics (Cambridge University Press, Cambridge, 1995).

[37] R. A. Bartels, A. Paul, H. Green, H. C. Kapteyn, M. M. Murnane, S. Backus, I. P. Christov, Y. Liu, D. Attwood, and C. Jacobsen, Science 297, 376 (2002).

[38] P. Schmüser, M. Dohlus, and J. Rossbach, Ultraviolet and Soft X-ray Free-electron Lasers: Introduction to Physical Principles, Experimental Results, Technological Challenges (Springer, Berlin, 2008). 